\title{
MicroRNA-222-3p promotes tumor cell migration and invasion and inhibits apoptosis, and is correlated with an unfavorable prognosis of patients with renal cell carcinoma
}

\author{
LIWEN ZHAO $^{1,2^{*}}$, JING QUAN $^{1,2^{*}}$, ZUWEI LI $^{1,3}$, XIANG PAN $^{1,2}$, JINGYAO WANG $^{1}$, JINLING XU $^{1}$, \\ WEIJIE XU ${ }^{1}$, XIN GUAN $^{1}$, HANG LI $^{4}$, SHANGQI YANG ${ }^{4}$, YAOTING GUI ${ }^{1}$, YUN CHEN ${ }^{5}$ and YONGQING LAI ${ }^{1}$ \\ ${ }^{1}$ Guangdong and Shenzhen Key Laboratory of Male Reproductive Medicine and Genetics, Institute of Urology, \\ Peking University Shenzhen Hospital, Shenzhen PKU-HKUST Medical Center, Shenzhen, Guangdong 518036; \\ ${ }^{2}$ Department of Urology, Anhui Medical University, Hefei, Anhui 230032; ${ }^{3}$ Department of Urology, \\ Shantou University Medical College, Shantou, Guangdong 515041; Departments of ${ }^{4}$ Urology and \\ ${ }^{5}$ Ultrasound, Peking University Shenzhen Hospital, Shenzhen, Guangdong 518036, P.R. China
}

Received May 6, 2018; Accepted October 9, 2018

DOI: 10.3892/ijmm.2018.3931

\begin{abstract}
The aim of the present study was to investigate the role of microRNA (miR)-222-3p in renal cell carcinoma (RCC). The expression level of miR-222-3p was detected in RCC tissues and cell lines (ACHN, 786-O, Caki-1 and 769-P) and was identified to be significantly upregulated compared with the level in adjacent normal renal tissues and HK-2 cells. Further in vitro experiments demonstrated that the overexpression of miR-222-3p promoted the migration and invasion, and attenuated the apoptosis of 786-O cells, whereas the knockdown of miR-222-3p suppressed the migration and invasion and induced the apoptosis of 786-O cells. Similar results were observed in the ACHN cell line in terms of migration, invasion and apoptosis. Furthermore, the expression level of miR-222-3p was measured in 42 RCC formaldehyde-fixed paraffin-embedded samples, and the association between the expression of miR-222-3p and the pathological characteristics and overall survival rate of patients with RCC was analyzed. The results demonstrated that patients with a higher expression of miR-222-3p had a significantly lower overall survival rate, compared with those with a lower expression of miR-222-3p [hazard ratio $(\mathrm{HR})=5.120 ; \mathrm{P}=0.036]$. Multivariate analysis identified that patients with a higher expression of
\end{abstract}

Correspondence to: Professor Yongqing Lai, Guangdong and Shenzhen Key Laboratory of Male Reproductive Medicine and Genetics, Institute of Urology, Peking University Shenzhen Hospital, Shenzhen PKU-HKUST Medical Center, 1120 Lianhua Road, Shenzhen, Guangdong 518036, P.R. China

E-mail: yqlord@163.com

*Contributed equally

Key words: microRNA, microRNA-222-3p, renal cell carcinoma, biomarker, prognosis
miR-222-3p retained the statistically significant decrease in overall survival rate compared with patients with a lower expression of miR-222-3p $(\mathrm{HR}=5.636 ; \mathrm{P}=0.030)$. Furthermore, Kaplan-Meier survival curves indicated that patients with higher miR-222-3p had significantly lower overall survival rates compared with patients with lower miR-222-3p $(\mathrm{P}=0.020)$. Taken together, these results suggested that miR-222-3p serves as an onco-miR in RCC and may be a potential prognostic biomarker and therapeutic target in patients with RCC.

\section{Introduction}

Renal cell carcinoma (RCC) is the most common malignancy of the kidney and it is estimated that $\sim 65,340$ novel cases and 14,970 cases of mortality are likely to occur in the USA in 2018 (1). Clear cell RCC is the most common subtype, accounting for $\sim 70 \%$ all RCC cases (2). In total, $>25 \%$ patients have developed metastatic disease on presentation, and reliable biomarkers for screening RCC have not been established (3). $\mathrm{RCC}$ is resistant to conventional chemoradiotherapy, and surgery remains the primary form of curative treatment for localized RCC (4). Despite advances in diagnosis and treatment, the 5-year overall survival rate of RCC is <50\% (4). Therefore, novel diagnostic approaches and therapeutic strategies for RCC are urgently required.

MicroRNAs (miRs) are a class of small non-coding RNAs that suppress gene expression by binding to the 3'-untranslated region of mRNA at the post-transcriptional level (5). Accumulating evidence suggested that miRs may be important in the development of malignancies (6). Previous studies demonstrated that miRs are involved in a number of cancer cell biological processes, including metastasis (7), invasion (8), angiogenesis (9), proliferation, apoptosis (10), differentiation (11), metabolism (12) and drug resistance (13). The dysregulation of miRs is observed in the majority of types of cancer, including RCC (14). In previous studies, numerous miRs have been demonstrated to be involved in RCC, including miR-720 (15), miR-203 (16), miR-204-3p (17) and miR-32-5p (18). miR-222 is 
associated with tumorigenesis in gastric cancer (19), glioma (20) and lung cancer (21), among others; however, the role of miR-222 in RCC remains to be fully elucidated.

In the present study, the expression of miR-222-3p was investigated in clinical RCC samples and cell lines in order to determine its effects on the migration, invasion and apoptosis of RCC cells and to analyze its association with overall survival rates. The aim of the present study was to determine whether miR-222-3p may serve as a non-invasive prognostic biomarker and therapeutic target for patients with RCC.

\section{Materials and methods}

Clinical samples. Patients who were initially treated, without previous surgery, radiotherapy, chemotherapy and other adjuvant therapy were included in the present study. Patients who had been diagnosed with tumors other than renal cell carcinoma were excluded. Tumor tissues and corresponding normal renal tissues were obtained from 28 patients with RCC at the Department of Urology, Peking University Shenzhen Hospital (Shenzhen, China) from January 2013 to January 2016. The clinicopathological parameters are summarized in Table I. In addition, 42 formaldehyde-fixed paraffin-embedded (FFPE) RCC samples were obtained from the Department of Pathology, Peking University Shenzhen Hospital. The clinical and pathological characteristics are listed in Table II. Follow-up data was obtained for the 42 FFPE specimens; however, not for the 28 patients, as no the follow-up data were available for the 28 patients, which is a limitation of the study. The present study was approved by the Ethics Committee of Peking University Shenzhen Hospital, and informed consent was obtained from all the patients.

RNA isolation and reverse transcription (RT). Total RNA was isolated from the tissues and cells using TRIzol ${ }^{\circledR}$ reagent (Invitrogen; Thermo Fisher Scientific, Inc., Waltham, MA, USA) and purified using an RNeasy Maxi kit (Qiagen $\mathrm{GmbH}$, Hilden, Germany) according to the manufacturer's protocol. The miRs of the FFPE samples were separated using the miRNeasy FFPE kit (Qiagen $\mathrm{GmbH}$ ) according to the manufacturer's protocol. Subsequently, the concentration and quality of RNAs were measured using NanoDrop 2000/2000c (Thermo Fisher Scientific, Inc.). In total, $\sim 1 \mu \mathrm{g}$ RNA was used to synthesize cDNA using the miScript II RT kit (Qiagen $\mathrm{GmbH}$ ) and the reaction conditions were as follows: $37^{\circ} \mathrm{C}$ for $60 \mathrm{~min}, 95^{\circ} \mathrm{C}$ for $5 \mathrm{~min}$ and $4^{\circ} \mathrm{C}$ until completion.

$R T$-quantitative polymerase chain reaction ( $q P C R)$ analysis. The RT-qPCR analysis was performed with the miScript SYBR $^{\circledR}$ Green PCR kit (Qiagen GmbH). U6 served as an internal control. The reaction contained $0.5 \mu 1$ primer, $1 \mu \mathrm{l}$ cDNA, $5 \mu 1$ 2x QuantiTect SYBR Green PCR Master Mix and $3 \mu 1$ RNase-free water. The reaction was performed in the Roche Lightcycler 480 Real-Time PCR system (Roche Diagnostics, Basel, Switzerland) and the reaction conditions were as follows: $95^{\circ} \mathrm{C}$ for $2 \mathrm{~min}$, followed by 40 cycles of $95^{\circ} \mathrm{C}$ for $10 \mathrm{sec}, 55^{\circ} \mathrm{C}$ for $30 \mathrm{sec}$ and $72^{\circ} \mathrm{C}$ for $30 \mathrm{sec}$. The primers used in the experiment are summarized in Table III. The reference gene was U6. The relative expression of miR-222-3p was evaluated using the comparative $\mathrm{Cq}$ and analyzed using the $2^{-\Delta \Delta \mathrm{Cq}}$ method $(22,23)$.
Table I. Clinicopathological features in patients with renal cell carcinoma.

\begin{tabular}{lc}
\hline Characteristic & Cases, $\mathrm{n}$ \\
\hline Mean age, years (range) & $45(24-87)$ \\
Sex & \\
Male & 17 \\
Female & 11 \\
Tumor stage & \\
T1 & 17 \\
T2 & 4 \\
T3 + T4 & 7 \\
Fuhrman grade & \\
I & 12 \\
II & 8 \\
III & 7 \\
IV & 1 \\
AJCC clinical stage & \\
I & 15 \\
II & 3 \\
III+IV & 10 \\
\hline
\end{tabular}

AJCC, American Joint Committee on Cancer.

Cell culture and transfection. The 786-O, ACHN, 769-P and Caki-1 RCC cell lines and the HK-2 normal human renal tubular epithelial cell line were purchased from the American Type Culture Collection (Manassas, VA, USA). According to Cellosaurus (https://web.expasy.org/cellosaurus), the ACHN, 769P, 786-O and Caki-1 cell lines appear to be papillary renal cell carcinoma, renal cell carcinoma, renal cell carcinoma and clear cell renal cell carcinoma, respectively. The HK-2 cells were cultured in keratinocyte serum-free medium (ScienCell Research Laboratories, Inc., Carlsbad, CA, USA) supplemented with $1 \%$ keratinocyte growth supplement (ScienCell Research Laboratories, Inc.), with $100 \mu \mathrm{l} / \mathrm{ml}$ penicillin and $100 \mathrm{mg} / \mathrm{ml}$ streptomycin sulfate in a humidified incubator with a $5 \% \mathrm{CO}_{2}$ atmosphere at $37^{\circ} \mathrm{C}$. The ACHN and Caki-1 cells were cultured in Dulbecco's modified Eagle's medium (Gibco; Thermo Fisher Scientific, Inc.) and McCoy's 5A (Gibco; Thermo Fisher Scientific, Inc.) respectively, and supplemented with $10 \%$ fetal bovine serum (FBS, Gibco; Thermo Fisher Scientific, Inc.) and $1 \%$ antibiotics $(100 \mu \mathrm{l} / \mathrm{ml}$ penicillin and $100 \mathrm{mg} / \mathrm{ml}$ streptomycin sulfate). The 769-P and 786-O cells were cultured in the same manner in RPMI-1640 medium (Gibco; Thermo Fisher Scientific, Inc.). A quick cell mycoplasma rapid test kit (Shanghai Life iLAB Bio Technology Co., Ltd., Shanghai, China) was used to detect whether the cells were contaminated with mycoplasma, according to the manufacturer's protocol. The miR-222-3p (miRbase; http://www.mirbase.org/; accession no. MIMAT0000279) mimics, negative control (NC), miR-222-3p inhibitor and inhibitor negative control (NC; GenePharma Co., Ltd., Shanghai, China) were transfected into cells at a concentration of $50 \mathrm{nM}$ using Lipofectamine ${ }^{\circledR} 3000$ (Invitrogen; Thermo Fisher Scientific, Inc.). The sequences of miRNAs are listed in Table III. 
Table II. Association between miR-222-3p status and clinicopathologic variables in formaldehyde-fixed-paraffin-embedded renal cell carcinoma tissue samples.

\begin{tabular}{|c|c|c|c|c|}
\hline \multirow[b]{2}{*}{ Variable } & \multirow[b]{2}{*}{ Total, $\mathrm{n}$} & \multicolumn{2}{|c|}{ miR-222-3p status } & \multirow[b]{2}{*}{ P-value } \\
\hline & & High, $n$ & Low, $\mathrm{n}$ & \\
\hline Sex & & & & $0.525^{\mathrm{a}}$ \\
\hline Male & 26 & 14 & 12 & \\
\hline Female & 16 & 7 & 9 & \\
\hline Age, years & & & & $1.000^{\mathrm{b}}$ \\
\hline$\leq 60$ & 33 & 16 & 17 & \\
\hline$>60$ & 9 & 5 & 4 & \\
\hline Tumor size, $\mathrm{cm}$ & & & & $0.116^{\mathrm{a}}$ \\
\hline$\leq 4.0$ & 17 & 6 & 11 & \\
\hline$>4.0$ & 25 & 15 & 10 & \\
\hline Tumor stage & & & & $0.747^{\mathrm{a}}$ \\
\hline $\mathrm{I}+\mathrm{II}$ & 27 & 13 & 14 & \\
\hline III+IV & 15 & 8 & 7 & \\
\hline
\end{tabular}

miR-222-3p status cut-off point: median. ${ }^{\mathrm{a} C a l c u l a t e d}$ with Pearson's $\chi^{2}$ test. ${ }^{\mathrm{b}} \mathrm{Calculated}$ with Fisher's exact test. miR, microRNA.

Table III. Sequences of primers and miRs.

Primer/miR

miR-222-3p

U6

miR-222-3p mimic

miR-222-3p inhibitor

$\mathrm{NC}$

Inhibitor NC
Sequence

Forward: 5'-AGCTACATCTGGCTACTGGGT-3'

Reverse: Universal primers (miScript SYBR Green PCR kit)

Forward: 5'-CTCGCTTCGGCAGCACA-3'

Reverse: 5'-ACGCTTCACGAATTTGCGT-3'

Forward: 5'-AGCUACAUCUGGCUACUGGGU-3'

Reverse: 5'-CCAGUAGCCAGAUGUAGCUUU-3'

5'-ACCCAGUAGCCAGAUGUAGCU-3'

Forward: 5'-UUCUCCGAACGUGUCACGUTT-3'

Reverse: 5'-ACGUGACACGUUCGGAGAATT-3'

5'-CAGUACUUUUGUGUAGUACAA-3'

miR, microRNA; NC, negative control; PCR, polymerase chain reaction.

Wound healing assay. The RCC cells (ACHN and 786-O) were seeded into 6 -well plates $\left(3 \times 10^{5}\right.$ cells/well) and incubated in a humidified chamber supplemented with $5 \% \mathrm{CO}_{2}$. The cells were transfected with miR-222-3p mimics, inhibitor and corresponding NC when they reached $\sim 90 \%$ confluence. Following $6 \mathrm{~h}$ transfection, a wound was created using a sterile 200- $\mu \mathrm{l}$ pipette tip, followed by washing with PBS three times. Images were captured using a digital light microscope (magnification, $\mathrm{x} 100)$ at 0 and $24 \mathrm{~h}$ following introduction of the wound.

Cell migration and invasion assays. The cell migration and invasion capacities were evaluated using a Transwell assay. Chambers coated with Matrigel (BD Biosciences, Franklin Lakes, NJ, USA) were used for the invasion assay, whereas Matrigel was omitted for the migration assay. Following $24 \mathrm{~h}$ transfection, $\sim 100 \mu 1$ serum-free medium containing $3 \times 10^{4}$ cells was added to the upper chambers, and $500 \mu 1$ medium supplemented with $10 \%$ FBS was added to the lower chambers. The migration and invasion duration was 24 and $36 \mathrm{~h}$, respectively. Subsequently, the cells were fixed in $4 \%$ formaldehyde for $15 \mathrm{~min}$ at room temperature and stained with $0.1 \%$ crystal violet solution for $25 \mathrm{~min}$ at room temperature. Images of those cells that had migrated or invaded to the opposite side of the membrane were captured using a light microscope (Leica Microsystems GmbH, Wetzlar, Germany; magnification, x100).

Apoptosis assay. Flow cytometry was performed to determine the effect of miR-222-3p on cell apoptosis in vitro. The cells $\left(\sim 3 \times 10^{5}\right)$ were added to 6 -well plates and cultured in an incubator for $24 \mathrm{~h}$; subsequently, the cells were treated with miR-222-3p mimics, miR-222-3p inhibitor or corresponding NC. Another group of cells (blank group) was treated with 
A
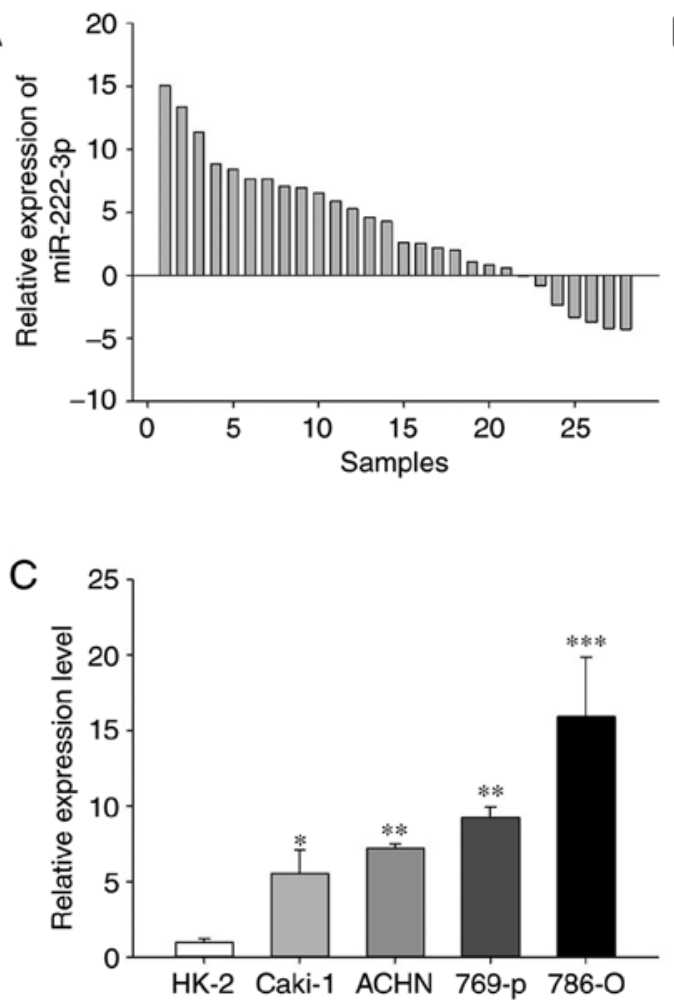

B

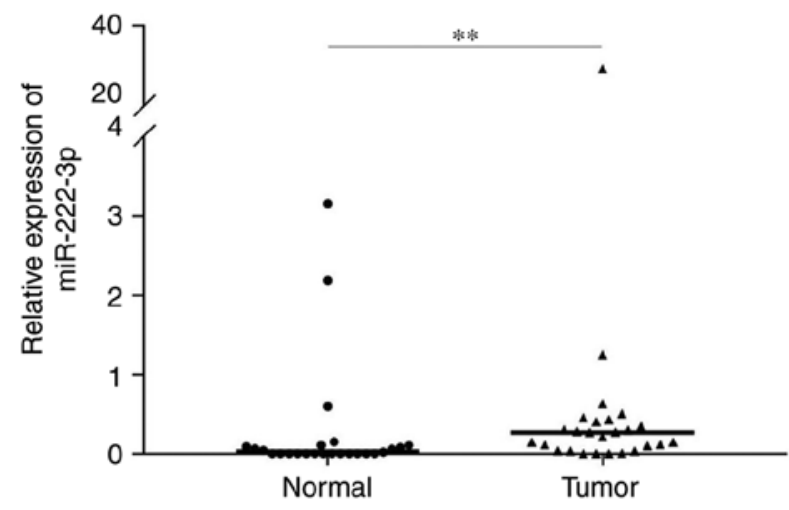

D

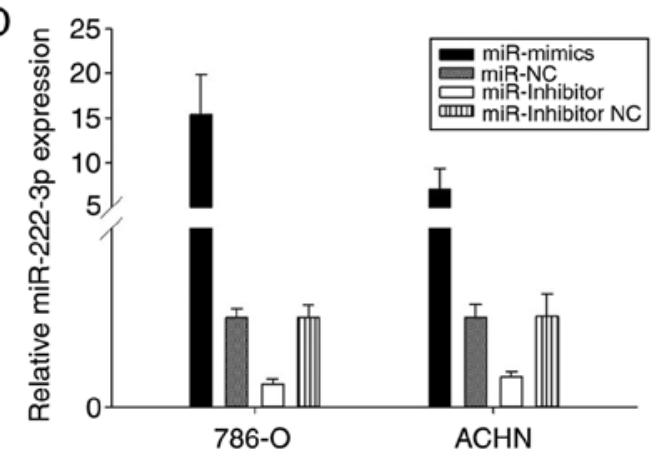

Figure 1. miR-222-3p is upregulated in RCC tissues and RCC cell lines. (A) Expression of miR-222-3p in 28 paired RCC tissues and adjacent normal renal tissues. (B) miR-222-3p was upregulated in RCC tissues compared with normal renal tissues. ${ }^{* *} \mathrm{P}<0.01$. (C) miR-222-3p was upregulated in RCC cell lines compared with HK-2 cells. ${ }^{*} \mathrm{P}<0.05,{ }^{* * *} \mathrm{P}<0.01$ and ${ }^{* * * *} \mathrm{P}<0.001$ vs. HK2 cells. (D) ACHN and $786-\mathrm{O}$ cells were treated with miR-222-3p mimics, NC, miR-222-3p inhibitor or inhibitor $\mathrm{NC}$ for $24 \mathrm{~h}$ and effects of these treatments were assessed in vitro. miR, microRNA; RCC, renal cell carcinoma; NC, negative control.

Lipofectamine ${ }^{\circledR} 3000$ but without mimics, inhibitor or corresponding NC. Following $48 \mathrm{~h}$ transfection, the cells were collected and washed twice with cold PBS, followed by staining with Annexin V-fluorescein isothiocyanate/propidium iodide (Invitrogen; Thermo Fisher Scientific, Inc.) according to the manufacturer's protocol. The apoptotic rate was detected using a flow cytometer (EPICS, Xl-4; Beckman Coulter, Inc., Brea, CA, USA) and analyzed using Kaluza Analysis 1.5a (Beckman Coulter, Inc.).

Clinical validation via the cancer genome atlas (TCGA) dataset. The correlation between the expression of miR-222-3p and the prognosis of patients with RCC was analyzed using a TCGA dataset in OncoLnc (www.oncolnc.org). OncoLnc is a tool website that links TCGA survival data to miRNA expression levels. In OncoLnc, the input term used was 'hsa-miR-222-3p' and the lower and upper percentile were set to 50. Subsequently, the correlation between the expression of miR-222-3p and the overall survival rate of the patients who provided the FFPE samples was analyzed and Kaplan-Meier curves were constructed.

Bioinformatics and target prediction analysis. Target prediction was performed for miR-222-3p with starBase v2.0 (http://starbase.sysu.edu.cn/browseIntersectTargetSite. php), PicTar (https://pictar.mdc-berlin.de), TargetScanHuman (www.targetscan.org) and PITA (https://genie.weizmann. ac.il/pubs/mir07/mir07_prediction.html). Only predictions by at least three programs were included in the analysis.
Statistical analysis. The results of the experiments were analyzed using SPSS 19.0 (IBM Corp., Armonk, NY, USA) and are presented as the mean \pm standard deviation or median. Differences between normal renal tissues and RCC tissues were statistically analyzed using non-parametric tests and presented as median values. The differences in the expression level of miR-222-3p among cell lines was statistically analyzed by one-way analysis of variance and Dunnett's post hoc test. Setting the median as a cutoff point, the expression level of miR-222-3p was classified into higher and lower groups. The association between the expression of miR-222-3p and clinical characteristics was evaluated by Pearson's $\chi^{2}$ test and Fisher's exact test. Kaplan-Meier overall survival curves were constructed to evaluate the effect of miR-222-3p on the prognosis of patients with x RCC. Differences between the curves were analyzed by the log-rank test. Univariate and multivariate Cox regression analyses were also performed. The overall survival was defined as the time between the first surgery for RCC and the patient succumbing to mortality from any cause. $\mathrm{P}<0.05$ was considered to indicate a statistically significant difference.

\section{Results}

miR-222-3p dysregulation in RCC. RT-qPCR analysis was performed to detect the expression levels of miR-222-3p in the RCC tissues and cell lines. As presented in Fig. 1, in the 28 paired tissues, miR-222-3p was significantly upregulated in the RCC tissues compared with the adjacent normal renal tissues (Fig. 1A and B). In addition, miR-222-3p was upregulated in 
A
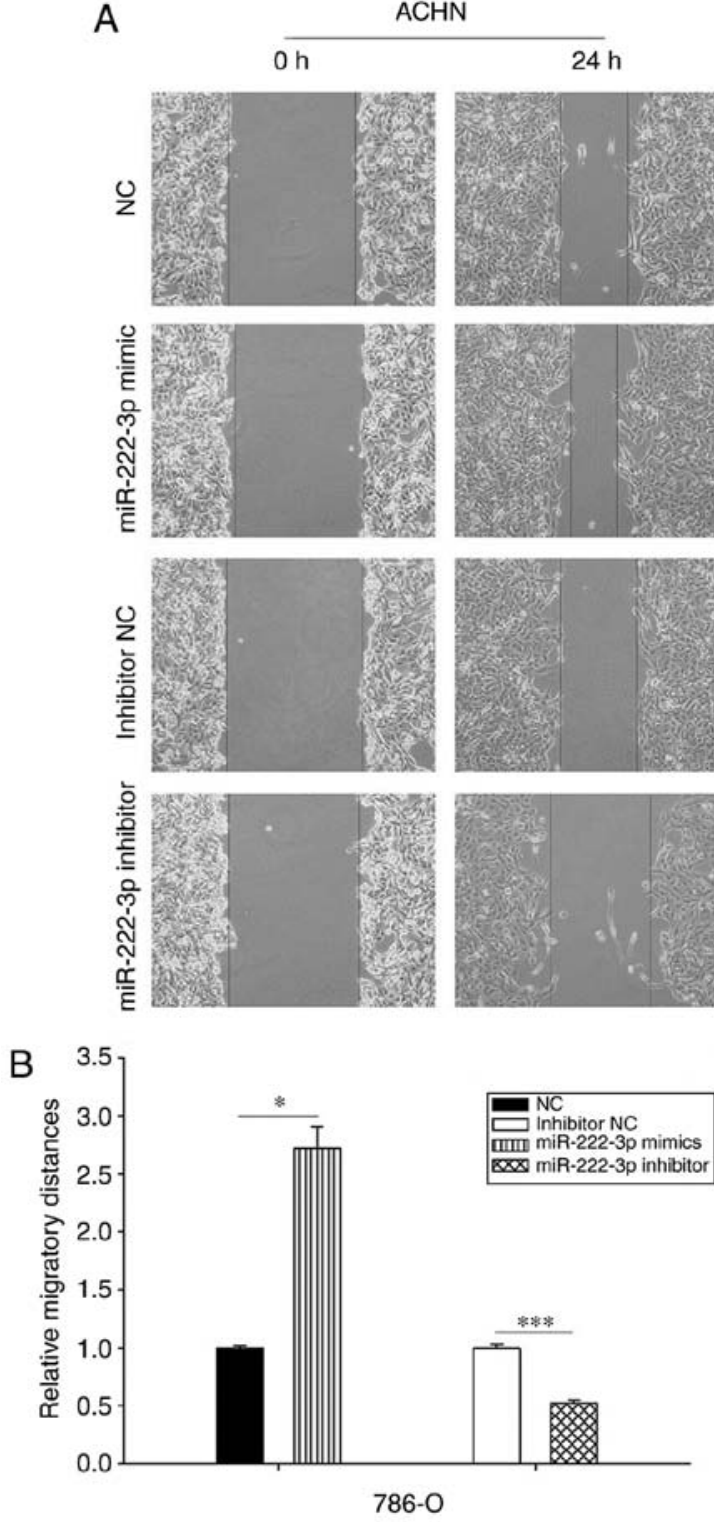

$24 \mathrm{~h}$
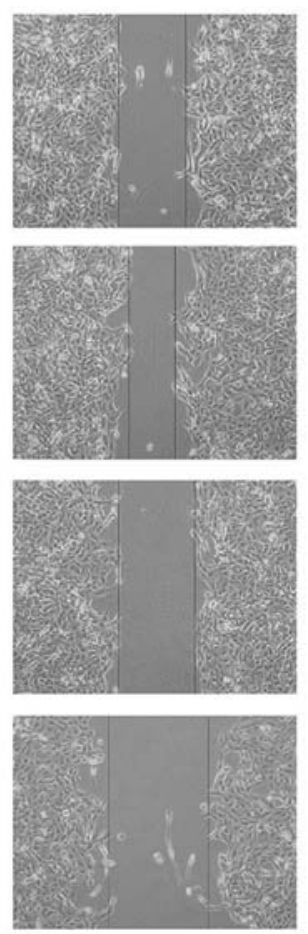

\section{C}

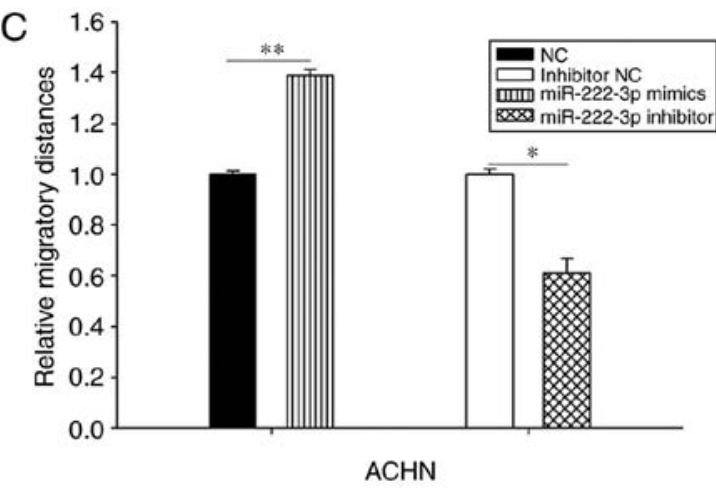

Figure 2. Results of wound healing assay. (A) Representative images of wound healing assays in ACHN and 786-O cells treated with miR-222-3p mimics, NC, miR-222-3p inhibitor or inhibitor NC (magnification, x100). (B) Overexpression of miR-222-3p enhanced 786-O cell migration, whereas knockdown of miR-222-3p inhibited 786-O cell migration. (C) Overexpression of miR-222-3p enhanced ACHN cell migration and knockdown of miR-222-3p inhibited ACHN cell migration. ${ }^{*} \mathrm{P}<0.05,{ }^{* * *} \mathrm{P}<0.01,{ }^{* * *} \mathrm{P}<0.001$. miR, microRNA; NC, negative control.

the RCC cell lines compared with the HK-2 cells (Fig. 1C). Therefore, the results suggested that miR-222-3p may serve as an onco-miR in RCC.

The 786-O and ACHN cell lines were selected for use in the subsequent experiments. The 786-O cells are RCC cells and ACHN cells are papillary RCC cells. By transfecting with miR-222-3p mimics, miR-222-3p inhibitor and corresponding NC, miR-222-3p was overexpressed or knocked down. The 24-h transfection efficiency was detected by RT-qPCR analysis (Fig. 1D).

miR-222-3p accelerates cell mobility. Wound healing and Transwell assays were performed to evaluate the effect of miR-222-3p on cell mobility. The results demonstrated that the overexpression of miR-222-3p promoted cell migration. As presented in Fig. 2, cells transfected with miR-222-3p mimics migrated faster compared with those transfected with
$\mathrm{NC}$; by contrast, cells transfected with miR-222-3p inhibitor migrated more slowly compared with those transfected with inhibitor NC. The results of the Transwell assay demonstrated that the migration ability of the two cell lines transfected with miR-222-3p mimics was enhanced compared with those transfected with NC, and the inhibition of miR-222-3p repressed cell migration ability compared with the inhibitor control group. In addition, the results of the Transwell assay demonstrated that the overexpression of miR-222-3p facilitated the invasion ability of cells compared with those in the NC group. The invasion ability of the two cell lines was suppressed in the miR-222-3p inhibitor group compared with the inhibitor NC group (Fig. 3). These results demonstrated that miR-222-3p promotes RCC cell migration and invasion in vitro.

miR-222-3p inhibits apoptosis in RCC cell lines. The results of the apoptosis assay demonstrated that the apoptotic rate of the 786-O 
A


$\mathrm{ACHN}$

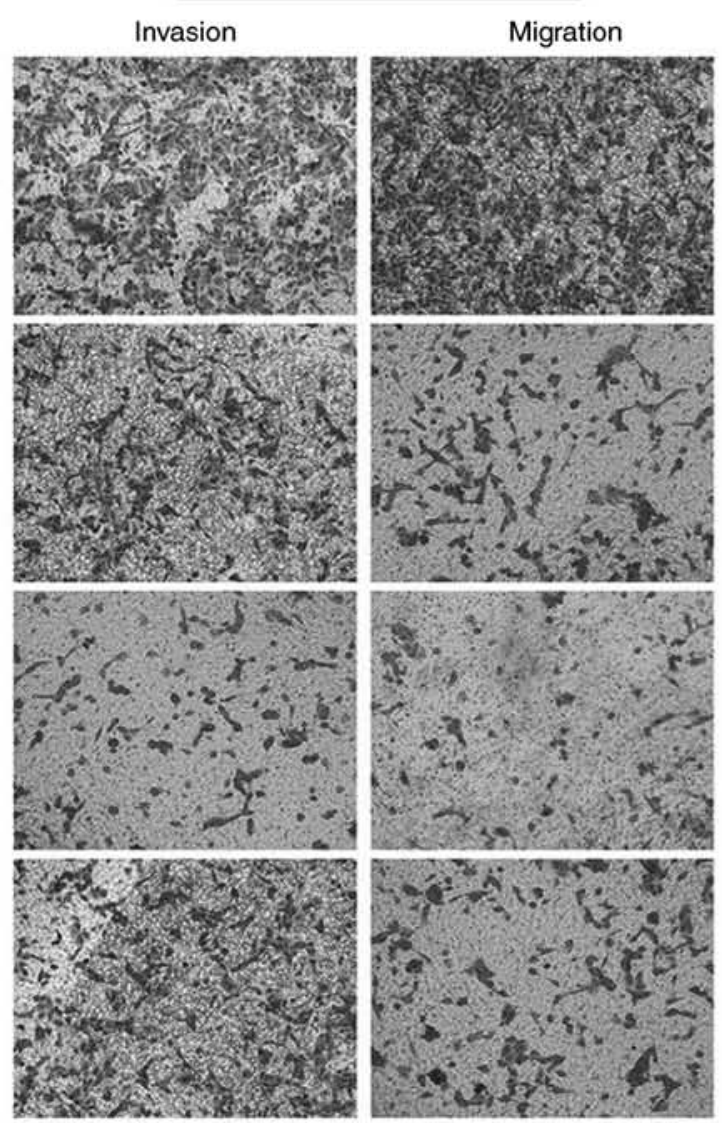

C
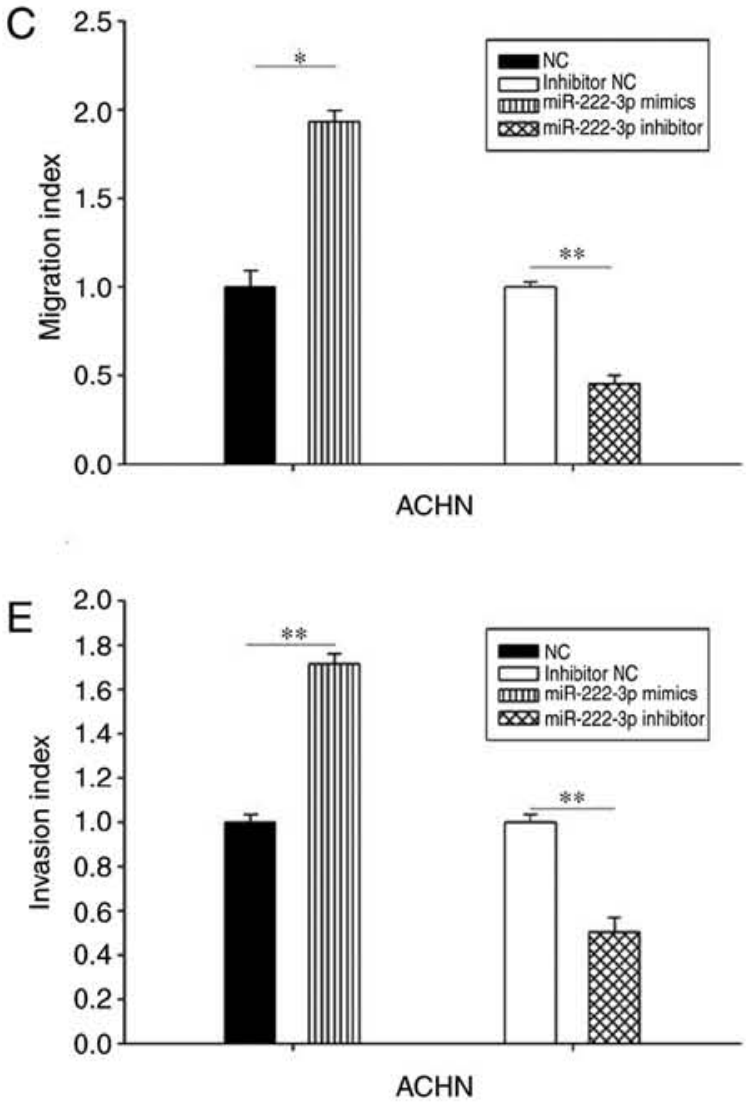

Figure 3. Results of Transwell assays. (A) Representative images of cells in the Transwell migration and invasion assays (magnification, x100). Overexpression of miR-222-3p enhanced (B) 786-O and (C) ACHN cell migration, whereas knockdown of miR-222-3p inhibited 786-O and ACHN cell migration. Overexpression of miR-222-3p enhanced (D) 786-O and (E) ACHN cell invasion, whereas knockdown of miR-222-3p inhibited 786-O and ACHN cell invasion. ${ }^{*} \mathrm{P}<0.05,{ }^{* *} \mathrm{P}<0.01,{ }^{* * *} \mathrm{P}<0.001 . \mathrm{miR}$, microRNA; $\mathrm{NC}$, negative control. 
A

NC $\quad 786-0$ miR-222-3p mimics
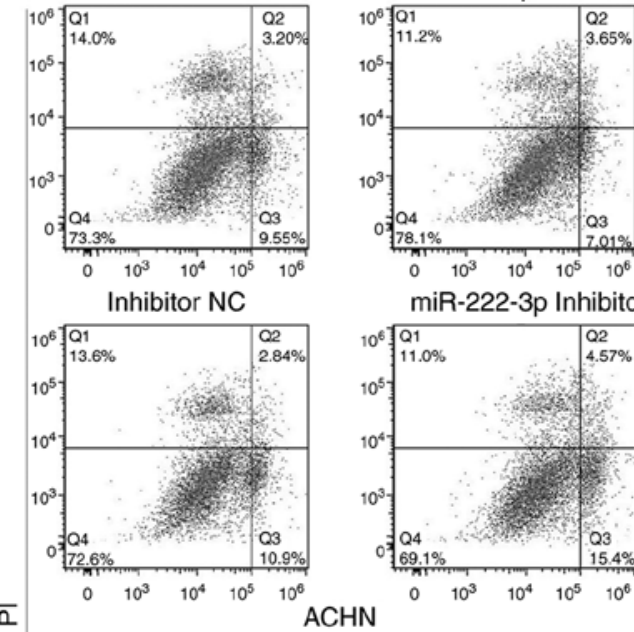

B
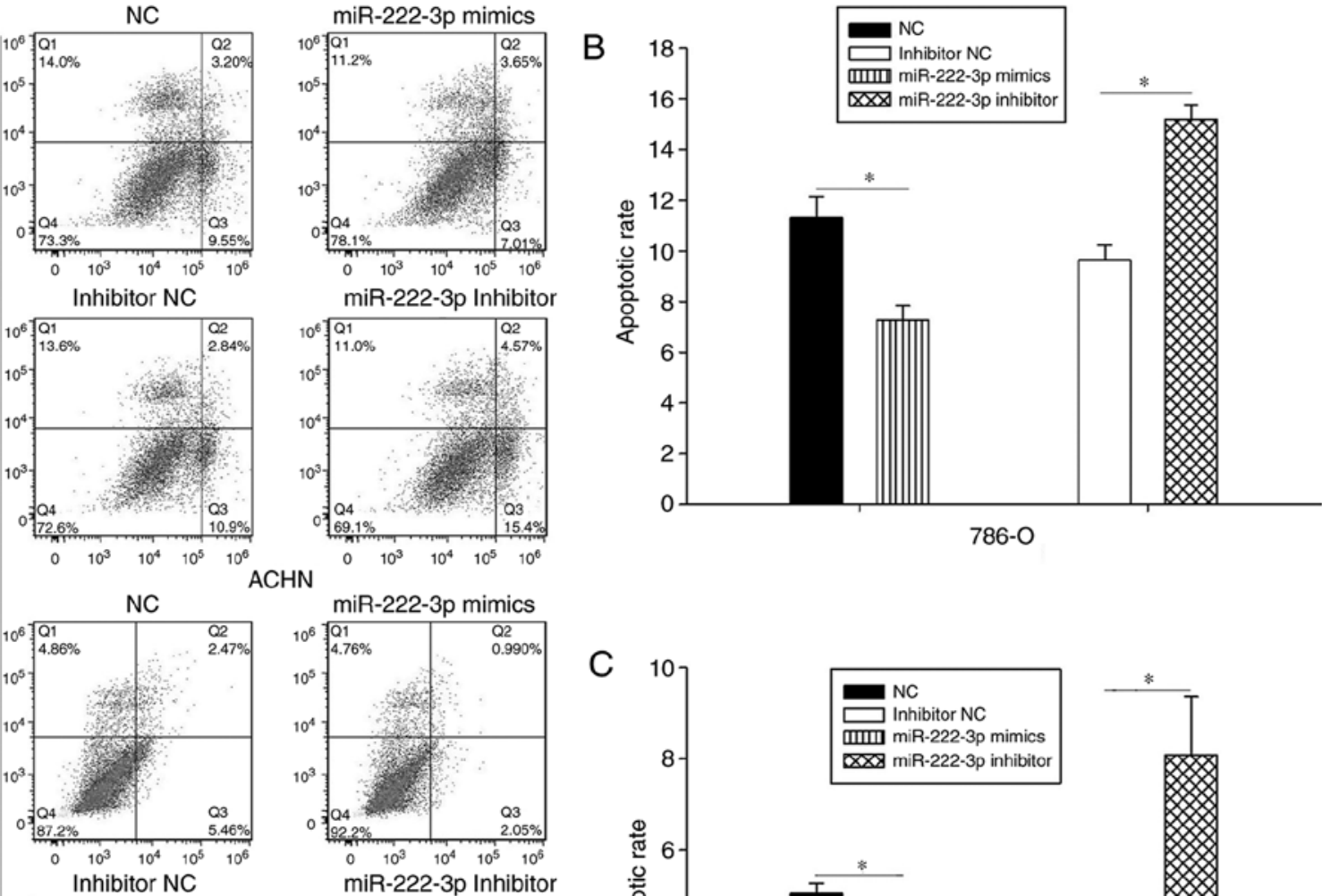

\section{.}
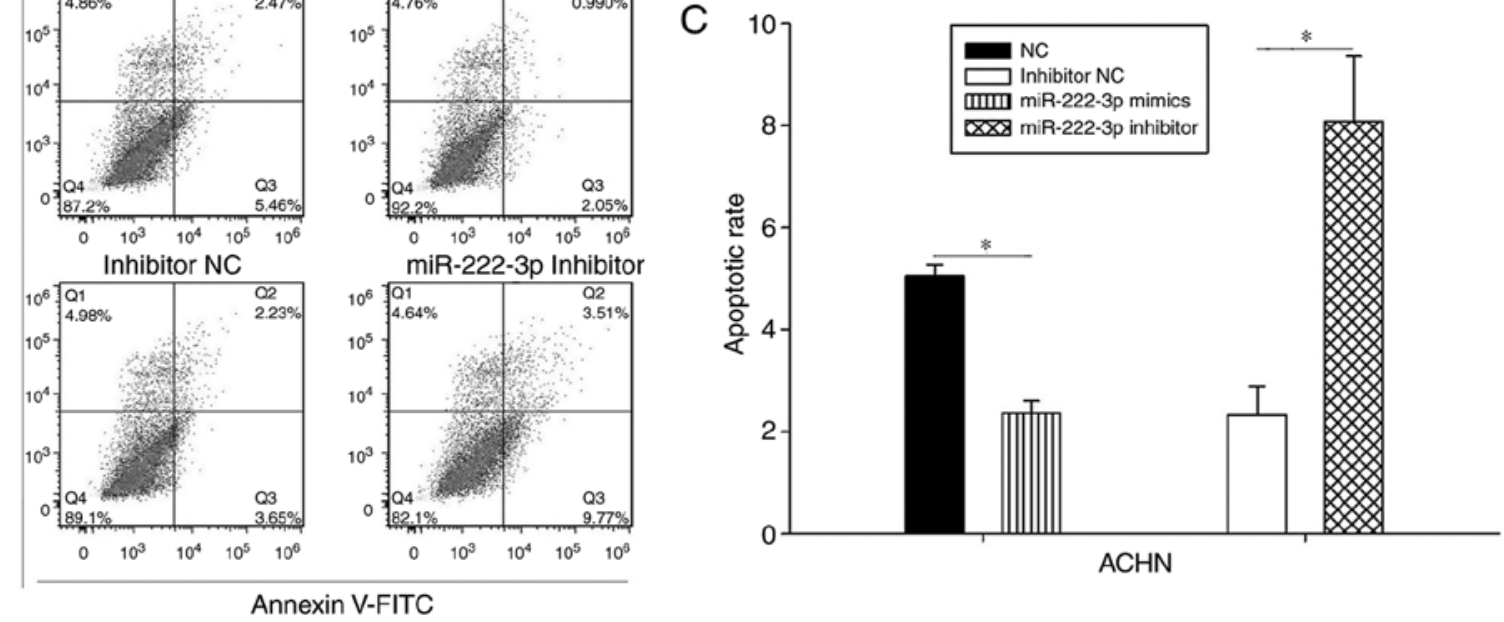

Figure 4. Results of apoptosis assay. Q3 represents the apoptotic rate. The rate was normalized with the blank group (data not shown). (A) Effect of miR-222-3p on the apoptosis of 786-O and ACHN cells. (B) Overexpression of miR-222-3p reduced 786-O cell apoptosis and knockdown of miR-222-3p promoted 786-O cell apoptosis. (C) Overexpression of miR-222-3p reduced ACHN cell apoptosis and knockdown of miR-222-3p promoted ACHN cell apoptosis. * $\mathrm{P}<0.05$. miR, microRNA; NC, negative control; PI, propidium iodide; FITC, fluorescein isothiocyanate; Q, quadrant.

cells was lower in the miR-222-3p mimics group compared with the NC group (Fig. 4A and B), with similar results observed in the ACHN cell line (Fig. 4A and C). The apoptotic rates of the two cell lines were higher in the inhibitor groups compared with those in the inhibitor NC group (Fig. 4). These results suggested that miR-222-3p partly decreased the apoptosis of RCC cells.

miR-222-3p is a potential prognostic marker for $R C C$. The expression level of miR-222-3p in 42 FFPE samples was detected by RT-qPCR analysis. No significant association was observed between the expression level of miR-222-3p and sex $(\mathrm{P}=0.525)$, age $(\mathrm{P}=1.000)$, tumor size $(\mathrm{P}=0.116)$ or tumor stage $(\mathrm{P}=0.747$; Table II). However, patients with a higher expression of miR-222-3p exhibited a statistically significant shorter overall survival rate, compared with patients with a lower expression of miR-222-3p [hazard ratio $(\mathrm{HR})=5.120$; $95 \%$ confidence interval $(\mathrm{CI})=1.113-23.539 ; \mathrm{P}=0.036]$. When controlling for age, sex, tumor size and tumor stage in the multivariate analysis, patients with a higher expression of miR-222-3p retained the statistically significant decrease in overall survival rate compared with patients with a lower expression of $\mathrm{miR}-222-3 \mathrm{p}(\mathrm{HR}=5.636$; 95\% CI=1.181-26.882; $\mathrm{P}=0.030$; Table IV). Furthermore, the Kaplan-Meier survival curves demonstrated that patients with higher expression levels of miR-222-3p exhibited significantly lower overall survival rates compared with patients with lower levels of miR-222-3p ( $\mathrm{P}=0.020$; Fig. 5). The results from the database analysis in OncoLnc of 506 RCC samples additionally demonstrated that patients with a higher expression of miR-222-3p exhibited significantly lower overall survival rates compared with patients with a lower expression of miR-222-3p $(\mathrm{P}<0.01$; Fig. 6). These results suggested that miR-222-3p may serve as a potential prognostic biomarker for patients with RCC.

Potential targets of $m i R-222-3 p$. In order to examine the potential involvement of miR-222-3p in the tumorigenesis of RCC, numerous databases were searched. The results demonstrated that 37 target genes were significantly correlated with miR-222-3p (Table V). These genes will be the focus of future investigations. 
Table IV. Expression of microRNR-222-3p and patient survival rates.

A, Univariate analysis

\begin{tabular}{lcc}
\hline & \multicolumn{2}{c}{ Overall survival } \\
\cline { 2 - 3 } Variable & HR $(95 \% \mathrm{CI})$ \\
\hline P-value & 1 & \\
\hline Low & & 0.036 \\
High & $5.120(1.113-23.539)$ & 0.066 \\
Age & $2.943(0.932-9.293)$ & 0.983 \\
Sex & $0.988(0.313-3.117)$ & 0.262 \\
Tumor size & $2.113(0.571-7.818)$ & 0.010 \\
Tumor stage & $4.872(1.462-16.241)$ & \\
\hline
\end{tabular}

$\mathrm{B}$, Multivariate analysis ${ }^{\mathrm{a}}$

Overall survival

Variable $\quad$ HR $(95 \% \mathrm{CI})$

P-value

\begin{tabular}{lll}
\hline Low & \multicolumn{1}{c}{1} & \\
High & $5.636(1.181-26.882)$ & 0.030 \\
Age & $2.502(0.513-12.202)$ & 0.257 \\
Sex & $1.630(0.341-7.788)$ & 0.540 \\
Tumor size & $1.316(0.341-5.076)$ & 0.690 \\
Tumor stage & $4.346(1.233-15.313)$ & 0.022 \\
\hline
\end{tabular}

${ }^{a}$ Adjusted for patient age, sex, tumor stage and tumor size. HR was estimated using the Cox proportional hazard regression model. HR, hazard ratio; CI, confidence interval of estimated HR.

\section{Discussion}

RCC is the most common type of renal malignancy and, despite advances in therapeutic approaches, its prognosis remains poor (24). Novel approaches in RCC diagnosis and treatment are required. miRs have been reported to be involved in a number of biological processes of tumorigenesis (6), and miR replacement therapy has been considered to be promising in cancer treatment (14).

Previous studies demonstrated that miR-222 is critical in the pathogenesis of a number of diseases. The upregulation of miR-222 in response to increased extracellular signal-regulated kinase $1 / 2$ activity exacerbates neointimal hyperplasia in diabetes mellitus (25). Zhao et al (26) demonstrated that long non-coding RNA Gas5 suppresses glioma malignancy by downregulating miR-222, and the knockdown of miR-222 was correlated with tumor size and survival rate in mice. A previous study performed by Tan et al (19) demonstrated that miR-222-3p promoted tumor cell proliferation and invasion and inhibited apoptosis by targeting homeodomain-interacting protein kinase 2 in gastric cancer. In colorectal cancer, miR-222 was identified to promote cell migration and invasion through targeting MIA3 (27). In addition, Zhang et al (28) observed

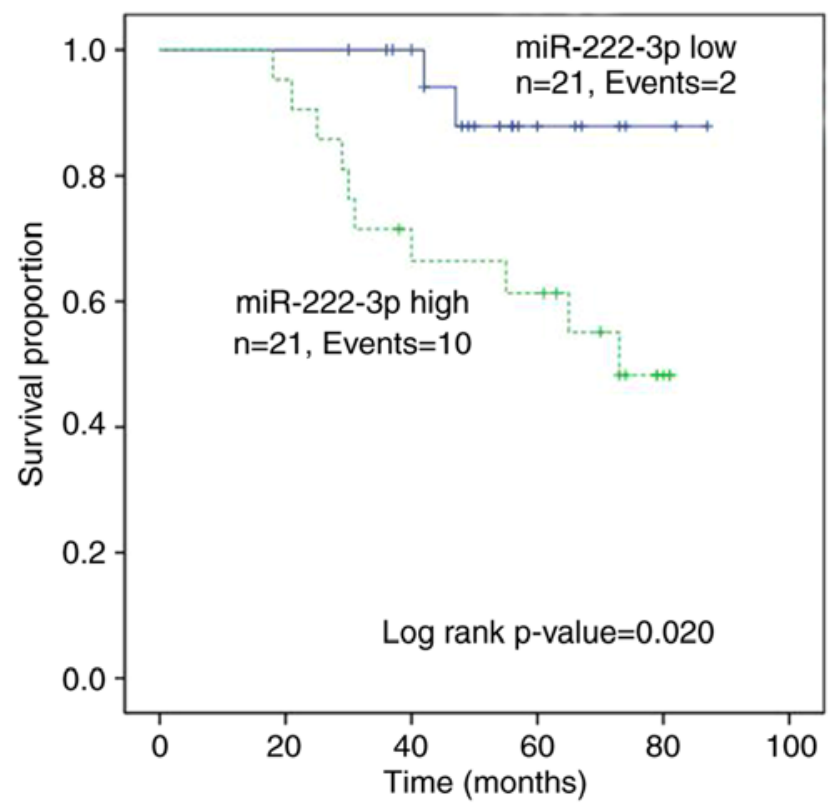

Figure 5. Survival rates of patients. Kaplan-Meier survival curves demonstrate a significantly improved prognosis of patients with tumors expressing lower levels of miR-222-3p in terms of overall survival rate. miR, microRNA.



Figure 6. Survival rates on database analysis. Kaplan-Meier curves showing the association between the expression of miR-222-3p and overall survival rate in The Cancer Genome Atlas dataset. Patients with lower levels of miR-222-3p exhibited significantly higher overall survival rates compared with those with higher expression levels of miR-222-3p. miR, microRNA.

that miR-222 inhibited tumor cell migration and invasion by downregulating guanine nucleotide binding protein, a inhibiting activity polypeptide 3 in hepatocellular carcinoma (28). Furthermore, miR-222 was identified to serve as a biomarker in a number of types of cancer, including lung (29), pancreatic (30), breast (31), bladder (32) and oral (33) cancer. miR-222 is involved in promoting cancer and suppressing cancer in different tumors, and its mechanism may be caused by different target genes in the downstream. According to Kafshdooz et al (34), overexpressed miRs may function as onco-miRs by downregulating tumor suppressor genes, whereas downregulated miRs may serve as tumor suppressors by negatively regulating oncogenes.

miR-222 has already been characterized as a discriminator miR for RCC subtypes (35) and miR-222-3p has been demonstrated to offer potential in distinguishing between normal tissues and RCC subtypes (36). However, in the present study, 
Table V. Target genes of microRNA-222-3p determined using starBase v2.0.

\begin{tabular}{|c|c|c|c|c|}
\hline No. & Gene & $\mathrm{R}$ & Rank & P-value \\
\hline 1 & SNX4 & -0.39727 & 15,268 & $<0.0001$ \\
\hline 2 & MYLIP & -0.38758 & 16,842 & $<0.0001$ \\
\hline 3 & PAIP2 & -0.34117 & 26,749 & $<0.0001$ \\
\hline 4 & PLEKHA2 & -0.30646 & 37,313 & $<0.0001$ \\
\hline 5 & CDKN1B & -0.30500 & 37,827 & $<0.0001$ \\
\hline 6 & PPP1R15B & -0.29684 & 40,824 & $<0.0001$ \\
\hline 7 & ARNT & -0.28919 & 43,721 & $<0.0001$ \\
\hline 8 & ANKHD1 & -0.21829 & 80,889 & 0.0001 \\
\hline 9 & ATXN1 & -0.16837 & 121,356 & 0.0034 \\
\hline 10 & FNDC3A & -0.14729 & 142,969 & 0.0106 \\
\hline 11 & POGZ & -0.14009 & 151,019 & 0.0152 \\
\hline 12 & MIA3 & -0.12970 & 163,365 & 0.0247 \\
\hline 13 & HIPK1 & -0.12861 & 164,708 & 0.0259 \\
\hline 14 & TMCC1 & -0.12025 & 175,357 & 0.0374 \\
\hline 15 & MIER3 & -0.11226 & 185,905 & 0.0521 \\
\hline 16 & MEGF9 & -0.09859 & 205,420 & 0.0883 \\
\hline 17 & RAB1A & -0.09235 & 214,787 & 0.1104 \\
\hline 18 & CTCF & -0.07860 & 236,696 & 0.1745 \\
\hline 19 & QKI & -0.07410 & 244,326 & 0.2006 \\
\hline 20 & HECTD2 & -0.06762 & 255,577 & 0.2429 \\
\hline 21 & MIDN & -0.06706 & 256,576 & 0.2469 \\
\hline 22 & VAPB & -0.03828 & 309,841 & 0.5089 \\
\hline 23 & TRPS 1 & -0.03086 & 324,412 & 0.5944 \\
\hline 24 & UBE2J1 & -0.03054 & 325,054 & 0.5983 \\
\hline 25 & ANKRD52 & -0.01265 & 361,316 & 0.8272 \\
\hline 26 & DYRK1A & 0.00371 & 379,893 & 0.9489 \\
\hline 27 & TCF12 & 0.00799 & 370,998 & 0.8904 \\
\hline 28 & DCUN1D1 & 0.00866 & 369,617 & 0.8812 \\
\hline 29 & TLE3 & 0.01128 & 364,187 & 0.8457 \\
\hline 30 & ARID1A & 0.04000 & 306,486 & 0.4901 \\
\hline 31 & BCL2L11 & 0.06746 & 255,858 & 0.2440 \\
\hline 32 & MEX3A & 0.07353 & 245,319 & 0.2041 \\
\hline 33 & $\mathrm{BMF}$ & 0.11406 & 183,484 & 0.0484 \\
\hline 34 & VGLL4 & 0.11958 & 176,213 & 0.0385 \\
\hline 35 & AMMECR1 & 0.16296 & 126,609 & 0.0047 \\
\hline 36 & ARF4 & 0.24646 & 63,505 & $<0.0001$ \\
\hline
\end{tabular}

$\mathrm{R}$ and $\mathrm{P}$-values were obtained using starBase v2.0. R, coefficient of correlation.

miR-222-3p was identified to promote the progression of RCC. These results demonstrated that miR-222 has an important function in RCC.

In the present study, miR-222-3p was observed to be upregulated in RCC tissues and cell lines, compared with adjacent normal renal tissues and the HK-2 cell line. The overexpression of miR-222-3p promoted cell migration and invasion and suppressed cellular apoptosis in RCC cell lines. Survival analysis demonstrated that a higher expression of miR-222-3p was correlated with poor prognosis in patients with RCC. However, a lack of target gene investigation was a limitation of the present study, which is to be performed in future investigations. Brodaczewska et al (37) demonstrated that RCC cells established from primary or metastatic disease may express different molecules or the same molecules; however, in different quantities. As 786-O and ACHN cells are different RCC cell lines, the apoptotic rate between the two cell lines was somewhat inconsistent. This may be caused by the different expression levels of apoptosis-associated proteins. However the exact mechanism leading to this difference remains to be fully elucidated, which was a limitation of the present study.

In conclusion, the present study demonstrated that miR-222-3p serves as an onco-miR in RCC, and a high expression of miR-222-3p was associated with poor prognosis in patients with RCC. These results suggested that miR-222-3p may serve as a biomarker and therapeutic target in patients with RCC. However, there were limitations to the present study, including the number of patients included being insufficient, and further investigations are required to elucidate the mechanism of miR-222-3p in RCC.

\section{Acknowledgements}

Not applicable.

\section{Funding}

The present study was supported by the Basic Research Project of Peking University Shenzhen Hospital (grant nos. JCYJ2017001, JCYJ2017004, JCYJ2017005, JCYJ2017006, JCYJ2017007 and JCYJ2017012; Shenzhen, China), the Clinical Research Project of Peking University Shenzhen Hospital (grant no. LCYJ2017001), the Science and Technology Development Fund Project of Shenzhen (grant no. JCYJ20170307111334308; Shenzhen, China) and the Clinical Research Project of Shenzhen Health Commission (grant no. SZLY2018023; Shenzhen, China).

\section{Availability of data and materials}

The analyzed datasets generated during the present study are available from the corresponding author on reasonable request.

\section{Authors' contributions}

YL, SY, YG and YC designed the experiments. LZ, JQ, ZL, $\mathrm{XP}$ and JW performed the experiments, and analyzed and interpreted the data. JX, WX and XG collected the renal cell carcinoma specimens and follow-up data of formaldehye-fixed paraffin-embedd specimens. HL analyzed the experimental data and wrote the manuscript. All authors read and approved the final version of the manuscript.

\section{Ethics approval and consent to participate}

The present study was approved by the Ethics Committee of Peking University Shenzhen Hospital (Shenzhen, China), and written informed consent for the use of the specimens for investigation purposes was provided by all patients prior to surgery. 


\section{Patient consent for publication}

Not applicable.

\section{Competing interests}

The authors declare that they have no competing interests.

\section{References}

1. Siegel RL, Miller KD and Jemal A: Cancer statistics, 2018. CA Cancer J Clin 68: 7-30, 2018.

2. Brugarolas J: Molecular genetics of clear-cell renal cell carcinoma. J Clin Oncol 32: 1968-1976, 2014.

3. Rossi SH, Klatte T, Usher-Smith J and Stewart GD: Epidemiology and screening for renal cancer. World J Urol 36: 1341-1353, 2018

4. Ljungberg B, Bensalah K, Canfield S, Dabestani S, Hofmann F Hora M, Kuczyk MA, Lam T, Marconi L, Merseburger AS, et al: EAU guidelines on renal cell carcinoma: 2014 update. Eur Urol 67: 913-924, 2015.

5. Karbasforooshan H, Roohbakhsh A and Karimi G: SIRT1 and microRNAs: The role in breast, lung and prostate cancers. Exp Cell Res 367: 1-6, 2018.

6. Kian R, Moradi S and Ghorbian S: Role of components of microRNA machinery in carcinogenesis. Exp Oncol 40: 2-9, 2018.

7. Fan Y, Ma X, Li H, Gao Y, Huang Q, Zhang Y, Bao X, Du Q, Luo G, Liu K, et al: miR-122 promotes metastasis of clear-cell renal cell carcinoma by downregulating Dicer. Int J Cancer 142: 547-560, 2018

8. Kabir TD, Ganda C, Brown RM, Beveridge DJ, Richardson KL, Chaturvedi V, Candy P, Epis M, Wintle L, Kalinowski F, et al: A microRNA-7/growth arrest specific 6/TYRO3 axis regulates the growth and invasiveness of sorafenib-resistant cells in human hepatocellular carcinoma. Hepatology 67: 216-231, 2018.

9. Fang L, Deng Z, Shatseva T, Yang J, Peng C, Du WW, Yee AJ, Ang LC, He C, Shan SW and Yang BB: MicroRNA miR-93 promotes tumor growth and angiogenesis by targeting integrin- $\beta 8$. Oncogene 30: 806-821, 2011.

10. Jia YJ, Liu ZB, Wang WG, Sun CB, Wei P, Yang YL, You MJ, Yu BH, Li XQ and Zhou XY: HDAC6 regulates microRNA-27b that suppresses proliferation, promotes apoptosis and target MET in diffuse large B-cell lymphoma. Leukemia 32: 703-711, 2018.

11. Ferreira AF, Calin GA, Picanco-Castro V, Kashima S, Covas DT and de Castro FA: Hematopoietic stem cells from induced pluripotent stem cells-considering the role of microRNA as a cell differentiation regulator. J Cell Sci 131: pii: jes203018, 2018

12. Kim S, Lee E, Jung J, Lee JW, Kim HJ, Kim J, Yoo HJ, Lee HJ, Chae SY, Jeon SM, et al: microRNA-155 positively regulates glucose metabolism via PIK3R1-FOXO3a-cMYC axis in breast cancer. Oncogene 37: 2982-2991, 2018

13. Hu W, Tan C, He Y, Zhang G, Xu Y and Tang J: Functional miRNAs in breast cancer drug resistance. Onco Targets Ther 11: 1529-1541, 2018.

14. Hosseinahli N, Aghapour M, Duijf PHG and Baradaran B: Treating cancer with microRNA replacement therapy: A literature review. J Cell Physiol 233: 5574-5588, 2018.

15. Bhat NS, Colden M, Dar AA, Saini S, Arora P, Shahryari V, Yamamura S, Tanaka Y, Kato T, Majid S and Dahiya R: MicroRNA-720 regulates E-cadherin- $\alpha$ E-catenin complex and promotes renal cell carcinoma. Mol Cancer Ther 16: 2840-2848, 2017.

16. Dasgupta P, Kulkarni P, Majid S, Shahryari V, Hashimoto Y, Bhat NS, Shiina M, Deng G, Saini S, Tabatabai ZL, et al: MicroRNA-203 inhibits long noncoding RNA HOTAIR and regulates tumorigenesis through epithelial-to-mesenchymal transition pathway in renal cell carcinoma. Mol Cancer Ther 17: 1061-1069, 2018

17. Han Z, Zhang Y, Sun Y, Chen J, Chang C, Wang X and Yeh S: ER $\beta$-mediated alteration of circATP2B1 and miR-204-3p signaling promotes invasion of clear cell renal cell carcinoma. Cancer Res 78: 2550-2563, 2018.
18. Wang M, Sun Y, Xu J, Lu J, Wang K, Yang DR, Yang G, Li G and Chang C: Preclinical studies using miR-32-5p to suppress clear cell renal cell carcinoma metastasis via altering the miR-32-5p/TR4/HGF/Met signaling. Int J Cancer 143: 100-112, 2018.

19. Tan X, Tang H, Bi J, Li N and Jia Y: MicroRNA-222-3p associated with Helicobacter pylori targets HIPK2 to promote cell proliferation, invasion, and inhibits apoptosis in gastric cancer. J Cell Biochem 119: 5153-5162, 2018.

20. Li Q, Shen K, Zhao Y, He X, Ma C, Wang L, Wang B, Liu J and Ma J: MicroRNA-222 promotes tumorigenesis via targeting DKK2 and activating the Wnt/ $\beta$-catenin signaling pathway. FEBS Lett 587: 1742-1748, 2013

21. Yamashita R, Sato M, Kakumu T, Hase T, Yogo N, Maruyama E, Sekido Y, Kondo M and Hasegawa Y: Growth inhibitory effects of miR-221 and miR-222 in non-small cell lung cancer cells. Cancer Med 4: 551-564, 2015.

22. Schmittgen TD and Livak KJ: Analyzing real-time PCR data by the comparative C(T) method. Nat Protoc 3: 1101-1108, 2008

23. Livak KJ and Schmittgen TD: Analysis of relative gene expression data using real-time quantitative PCR and the 2(-Delta Delta C(T)) method. Methods 25: 402-408, 2001.

24. Barata PC and Rini BI: Treatment of renal cell carcinoma: Current status and future directions. CA Cancer J Clin 67: 507-524, 2017.

25. Lightell DJ Jr, Moss SC and Woods TC: Upregulation of miR-221 and -222 in response to increased extracellular signal-regulated kinases $1 / 2$ activity exacerbates neointimal hyperplasia in diabetes mellitus. Atherosclerosis 269: 71-78, 2018

26. Zhao X, Wang P, Liu J, Zheng J, Liu Y, Chen J and Xue Y: Gas5 exerts tumor-suppressive functions in Human Glioma cells by targeting miR-222. Mol Ther 23: 1899-1911, 2015.

27. Gao H, Cong X, Zhou J and Guan M: MicroRNA-222 influences migration and invasion through MIA3 in colorectal cancer. Cancer Cell Int 17: 78, 2017.

28. Zhang Y, Yao J, Huan L, Lian J, Bao C, Li Y, Ge C, Li J, Yao M, Liang L and He X: GNAI3 inhibits tumor cell migration and invasion and is post-transcriptionally regulated by miR-222 in hepatocellular carcinoma. Cancer Lett 356: 978-984, 2015.

29. Lv S, Xue J, Wu C, Wang L, Wu J, Xu S, Liang X and Lou J: Identification of A panel of serum microRNAs as biomarkers for early detection of lung adenocarcinoma. J Cancer 8: 48-56, 2017.

30. Lee C, He H, Jiang Y, Di Y, Yang F, Li J, Jin C and Fu D: Elevated expression of tumor miR-222 in pancreatic cancer is associated with Ki67 and poor prognosis. Med Oncol 30: 700, 2013.

31. Han SH, Kim HJ, Gwak JM, Kim M, Chung YR and Park SY: MicroRNA-222 expression as a predictive marker for tumor progression in hormone receptor-positive breast cancer. J Breast Cancer 20: 35-44, 2017.

32. Puerta-Gil P, Garcia-Baquero R, Jia AY, Ocaña $S$, Alvarez-Múgica M, Alvarez-Ossorio JL, Cordon-Cardo C, Cava $\mathrm{F}$ and Sánchez-Carbayo M: miR-143, miR-222, and miR-452 are useful as tumor stratification and noninvasive diagnostic biomarkers for bladder cancer. Am J Pathol 180: 1808-1815, 2012

33. Chang YA, Weng SL, Yang SF, Chou $\mathrm{CH}$, Huang WC, Tu SJ, Chang TH, Huang CN, Jong YJ and Huang HD: A Three-MicroRNA signature as a potential biomarker for the early detection of oral cancer. Int J Mol Sci 19: pii: E758, 2018.

34. Kafshdooz L, Pourfathi H, Akbarzadeh A, Kafshdooz T, Razban Z, Sheervalilou R,Ebrahimi Sadr N, Khalilov R, Saghfi S, Kavetskyy T, et al: The role of microRNAs and nanoparticles in ovarian cancer: A review. Artif Cells Nanomed Biotechnol: 1-7, 2018 (Epub ahead of print).

35. Meo AD, Saleeb R, Wala SJ, Khella HW, Ding Q, Zhai H, Krishan K, Krizova A, Gabril M, Evans A, et al: A miRNA-based classification of renal cell carcinoma subtypes by PCR and in situ hybridization. Oncotarget 9: 2092-2104, 2017.

36. Youssef YM, White NM, Grigull J, Krizova A, Samy C, Mejia-Guerrero S, Evans A and Yousef GM: Accurate molecular classification of kidney cancer subtypes using microRNA signature. Eur Urol 59: 721-730, 2011.

37. Brodaczewska KK, Szczylik C, Fiedorowicz M, Porta C and Czarnecka AM: Choosing the right cell line for renal cell cancer research. Mol Cancer 15: 83, 2016. 